\title{
Shining new light on the motion of eutectic droplets across surfaces: A PEEM study of PtGe on Ge(110)
}

\author{
Bene Poelsema, Zhiguo Zhang $\odot$, Junia S. Solomon $\odot$, Harold J. W. Zandvliet $\odot$, and Arie van Houselt $\odot$ \\ Physics of Interfaces and Nanomaterials, MESA ${ }^{+}$Institute for Nanotechnology, \\ University of Twente, P.O. Box 217, 7500AE Enschede, The Netherlands
}

(Received 25 June 2021; revised 7 October 2021; accepted 7 December 2021; published 30 December 2021)

\begin{abstract}
Thermally stimulated motion of micron-sized eutectic PtGe droplets on Ge(110) has been studied in situ mainly by photoemission electron microscopy, low-energy electron microscopy, and spatially resolved lowenergy electron diffraction. In line with earlier studies of eutectic $\mathrm{AuSi}$, PtSi, AuGe, and PtGe clusters on, respectively, various $\mathrm{Si}$ and Ge substrates we find that the motion toward regions at higher temperature is driven by the entropy gain of substrate atoms which become constituents of the droplet during its journey. At $\sim 1100 \mathrm{~K}$, i.e., well above the bulk eutectic temperature, the direction is governed solely by the local thermal gradient, irrespective of eventual crystalline preferences. Access to the diffusivity of the host material (in this case $\mathrm{Ge}$ ) inside the eutectic droplets shows that this is well above one order of magnitude higher than expected if it was rate limiting for the velocity of the moving droplets. This excludes a significant gradient of the $(\mathrm{Ge})$ concentration inside the droplet and disqualifies dissolution-diffusion-deposition flow as the driving force for motion of the droplets on the surface, as assumed widely hitherto, to explain surface diffusion of eutectic droplets on surfaces. In addition, the interface between the droplet and the surface appears flat and we find no indication for "endotaxy." The droplets make direct contact with the flat Ge substrate through atomic steps, which are abundantly present at the interface. The droplets are surrounded by a $\mathrm{PtGe}_{3}$ wetting layer with an ordered $(2 \times$ 1) structure. Dissolution of the edges of the wetting layer at the leading edge of the droplet with an activation energy of $2.2 \mathrm{eV}$ is identified as the rate limiting step for its motion.
\end{abstract}

DOI: 10.1103/PhysRevMaterials.5.125602

\section{INTRODUCTION}

The motion of metal containing eutectic droplets inside a Si crystal subjected to a strong thermal gradient has been studied in detail by Cline and Anthony [1]. The droplets move toward higher temperature within the host material (Si). Subsequently, Si dissolves at the advancing side, diffuses through the eutectic, and is expelled and redeposited at the receding side. The migration speed was found to increase substantially at temperatures far above the eutectic temperature due to higher atomic percentages of the host material inside the droplet. Considerable attention has been paid to the dynamics of metallic droplets on surfaces since then. This includes the motion of Ga droplets on GaAs under incongruent evaporation conditions [2,3], thermomigration of PtSi droplets on stepped $\mathrm{Si}(111)$ and $\mathrm{Si}(100)$ [4-6], AuSi- and AuGe- droplets on various $\mathrm{Si}$ and $\mathrm{Ge}$ substrates [7,8], AuGe on $\mathrm{Ge}(110)$ [9], and, recently, electromigration of AuGe [10]. The surface studies were motivated by interest in possibilities for bottom-up fabrication of nanostructures, catalysis of standing up [11] and lying down nanowires $[12,13]$ on surfaces, and, last but not least, genuine interest in the complex physics of the motion of metallic droplets on surfaces. Although consensus is emerging on the role of the gain of entropy when the droplets travel toward hotter regions on the surface as the driving force for droplet mobility, many questions remain still open, such as the exact role of the atomic steps at the surface; the nature of the enhanced uniform morphology in the wake, i.e., in the trail behind the moving droplet; the morphology of the interface between the spherical droplet segments and the hosting surface; and the directionality and velocity of the mobile droplet segment and their influence on the size of the droplets. It is generally accepted that during their motion the droplets stay at the liquidus line and are in thermal equilibrium with the substrate. The current, quite commonly accepted, notion is that the diffusivity of the host material inside the eutectic droplets is rate limiting for their diffusion speed on the surface [4-6,9].

In our present contribution for micron-sized droplets on $\mathrm{Ge}(110)$ we critically evaluate the applicability of the Cline-Anthony (dissolution-diffusion-repulsion) mechanism for surface diffusion. We find no evidence for a driving role of atomic steps in the droplet motion. Moreover, most importantly, we find no indication at all for a linear dependence of the diffusion speed on the local thermal gradient at the surface as required in the Cline-Anthony model for particles with a constant size. We find clear evidence that the dissolution of the wetting layer at the leading edge of the droplet is the rate limiting step of the motion of the droplet.

\section{EXPERIMENT}

The photoemission electron microscopy (PEEM) and lowenergy electron microscopy (LEEM) experiments have been carried out with an Elmitec LEEM III instrument with a base pressure of $10^{-10} \mathrm{mbar}$. In PEEM, the surface was illuminated with a $100 \mathrm{~W}$ mercury discharge lamp $(\lambda=0.253 \mu \mathrm{m})$ incident at $16^{\circ}$ from the surface plane. The absolute temperature 


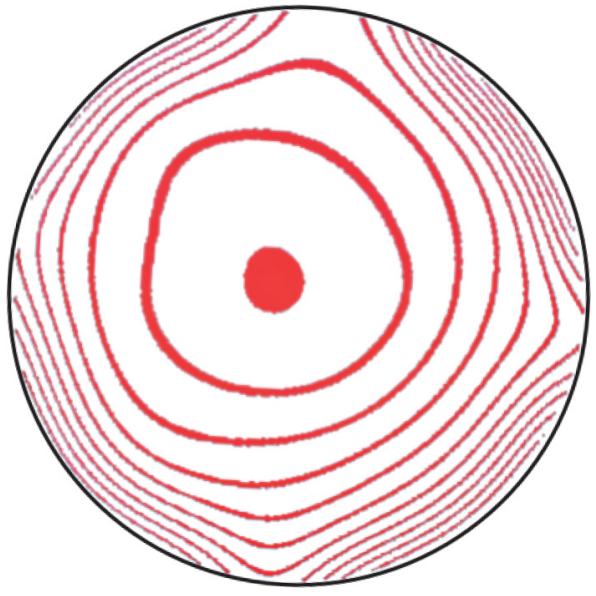

FIG. 1. Map of the isotherms on the Ge(110) substrate with a diameter of $7 \mathrm{~mm}$. The distance between the isotherms is $7 \mathrm{~K}$ and the maximum temperature in the center is $\sim 1100 \mathrm{~K}$.

reading is estimated to be correct within ca. $25 \mathrm{~K}$ and is calibrated by making the highly reasonable assumption that the eutectic temperature at the surface equals that of the bulk $(1050 \mathrm{~K}$ for $\mathrm{GePt})$. A Ge(110) substrate, $10 \mathrm{~mm} \times 10 \mathrm{~mm}$ nominally flat, single side polished $n$-type Ge (110) crystals (MTI Corporation, $R>50 \Omega \mathrm{cm}$ ) has been degassed for about $24 \mathrm{~h}$ at $700 \mathrm{~K}$, followed by several cycles of argon ion bombardment and flash annealing by $e$-beam bombardment at a temperature exceeding $1000 \mathrm{~K}$. Subsequently, Pt is deposited from a resistively heated $\mathrm{W}$ wire wrapped with high-purity (99.995\%) Pt (Alfa Aesar). The structure of the clean surface has been examined using low-energy electron diffraction (LEED). Like the findings in Ref. [14], we also observed a $c$ $(8 \times 10)$ structure at lower substrate temperatures, while above the Ge-Pt eutectic temperature only the nonreconstructed $(1 \times 1)$ structure is observed. With the sample kept at high temperatures for many hours a spectral analysis of a photo image has been obtained and the resulting temperature profile of the surface is shown in Fig. 1. The temperature is maximal in the center and falls off about $50 \mathrm{~K}$ toward the edges due mainly to heat conduction to the sample holder. This results in modest but persistent temperature gradients even quite close to the center of the substrate.

In the relevant area, the obtained temperature profile closely follows a paraboloid with $T(K)=1100-0.9 r^{2}(\mathrm{~mm})$ with $r$ being the distance from the center. Obviously, the temperature gradient increases linearly with $r$.

\section{RESULTS}

An amount equivalent to about five monolayers of $\mathrm{Pt}$ has been deposited with the $\mathrm{Ge}(110)$ substrate held at room temperature. Following this exposure, the crystal was heated slowly for several hours until a steady state set in with a maximum temperature of $\sim 1100 \mathrm{~K}$. Upon passing the eutectic temperature during heating a phase transition is observed in which the Ge-Pt clusters become liquid and get brighter than their environment in PEEM images and the larger droplets become mobile. Let us now consider the scenario for the motion of individual eutectic droplets in more detail. For this purpose, we seek guidance from the bulk phase diagram for Ge-Pt. The Ge-rich part of the phase diagram [15] has been reproduced in Fig. 2(a). Upon heating, the first eutectic solution emerges at $1050 \mathrm{~K}$. Above the eutectic temperature the droplet is in equilibrium with the supporting $\mathrm{Ge}(110)$ substrate. The shape and position of the liquidus line in the phase diagram provide information on the composition of the eutectic solution and the latent heat as a function of temperature. Let us consider a GePt droplet in equilibrium with the supporting $\mathrm{Ge}(110)$ substrate. The droplet-substrate combination consists of a constant number of particles at a constant pressure. Then Maxwell's equation $(\partial G / \partial T)_{N, p}=-S$ dictates that the total Gibbs free energy for this system decreases with increasing temperature. Consequently, the droplet moves toward the location with the highest temperature in the presence of a temperature gradient at the surface. During its trip the droplet with a constant number of $\mathrm{Pt}$ atoms accommodates additional $\mathrm{Ge}$ atoms originating from the substrate in order to keep fulfilling the composition condition imposed by the liquidus line [red curve in Fig. 2(a)].

The Gibbs free energy $g$ of a Ge atom in either the crystalline substrate or the liquid phase is by definition

$$
g_{x, l}=h_{x, l}-T s_{x, l},
$$

where the subscripts $x$ and $l$ refer to the crystalline and the liquid phase, respectively; $h$ is the enthalpy and $s$ is the entropy, both per Ge atom, and $T$ is the temperature. The Gibbs free energy per atom in the eutectic droplet also depends on the fraction of $\mathrm{Ge}$ atoms in the eutectic droplet $(\theta)$ [16],

$$
g_{l}(T, \theta)=g_{l}(T)-\Delta g_{\text {mix }}(T, \theta),
$$

where the $\Delta g_{\text {mix }}(T, \theta)$ is the mixing Gibbs free energy per $\mathrm{Ge}$ atom. In equilibrium, the Gibbs free energies per Ge atom in the crystalline phase and the eutectic droplet are the same, i.e., $g_{x}(T)=g_{l}(T, \theta)$. The means that in our system we have

$$
g_{x}(T) \neq g_{l}(T) .
$$

The Ge-particle currents from the substrate to the eutectic droplet and vice versa are then, by definition, given by

$$
\begin{aligned}
j_{x \rightarrow l} & =c n_{x} \exp \left[\frac{-\left(g_{b}-g_{x}\right)}{k_{B} T}\right] \\
\text { and } j_{l \rightarrow x} & =c n_{l} \exp \left[\frac{-\left(g_{b}-g_{l}\right)}{k_{B} T}\right],
\end{aligned}
$$

where $n_{x, l}$ refer to the Ge concentrations in the respective phases, $g_{b}$ is the activation barrier, and $c$ is a constant. With a relative density of Ge in the substrate equal to 1 and that of Ge in the droplet $\theta_{\mathrm{Ge}}$ given by

$$
\theta_{\mathrm{Ge}}=\frac{n_{l}}{n_{l}+n_{l, P t}},
$$

with $n_{l, P t}$ being the atomic concentration of $\mathrm{Pt}$ in the droplet, one obtains in equilibrium between droplet and substrate:

$$
\begin{aligned}
j_{x \rightarrow l} & =j_{l \rightarrow x}, \quad \text { or } \exp \left(\frac{g_{x}}{k_{B} T}\right)=\theta_{\mathrm{Ge}}, \exp \left(\frac{g_{l}}{k_{B} T}\right), \\
\text { or } \ln \theta_{\mathrm{Ge}} & =\frac{-\left(g_{l}-g_{x}\right)}{k_{B} T}=-\frac{\Delta g_{\mathrm{mix}}}{k_{B} T} .
\end{aligned}
$$



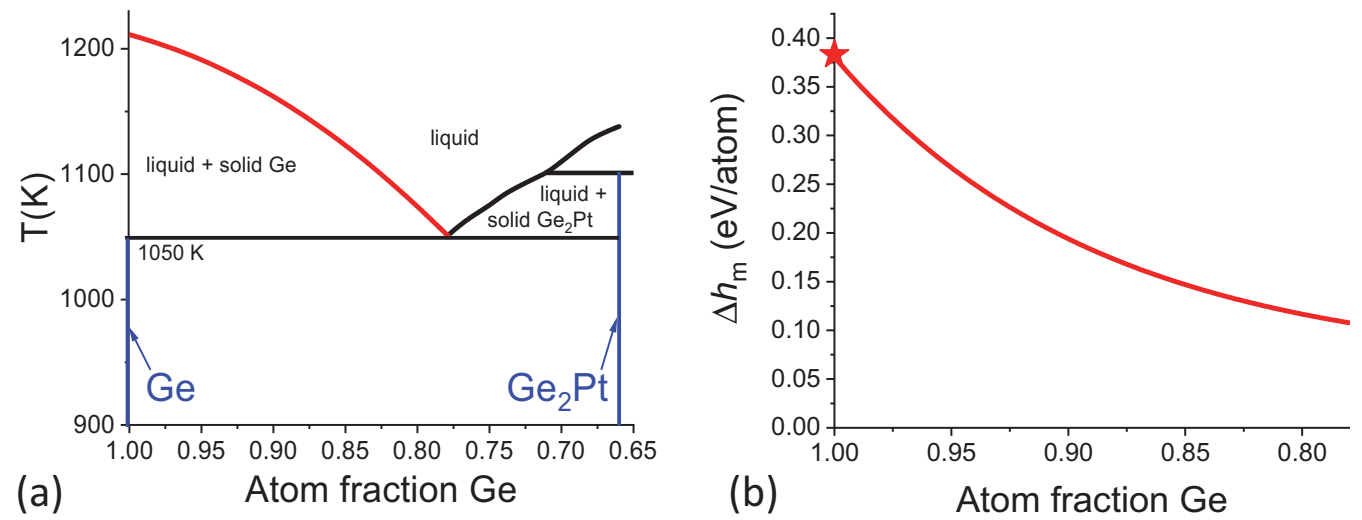

FIG. 2. (a) The high Ge content part of the GePt bulk phase diagram. At the eutectic temperature at 1050 K a eutectic phase, with a 78:22 at. \% Ge:Pt ratio, is formed in contact with pure $\mathrm{Ge}$ and $\mathrm{Ge}_{2} \mathrm{Pt}$. During the experiments the system moves along the red liquidus curve in (a). Data replotted from [15]. (b) Latent heat of fusion of Ge atoms within the eutectic droplet versus the atomic fraction of Ge within the droplet. The asterisk indicates the latent heat of pure Ge.

All expressions are exact. To acquire some more insight, we apply Eq. (1) and rewrite Eq. (6):

$$
\ln \theta_{\mathrm{Ge}}=\frac{\Delta h}{k_{B} T}-\frac{\Delta s}{k_{B}} .
$$

From a conventional analysis, i.e., from a plot of $\ln \vartheta_{\mathrm{Ge}}$ versus $1 / k_{B} T$, one derives naively $\Delta h_{m}$ as a function of $\vartheta_{\mathrm{Ge}}$. The result is shown in Fig. 2(b), which reveals a strong decay of the melting enthalpy with decreasing Ge content. However, a hidden $T$ dependence in the entropy term inevitably leads to an incorrect result for the melting enthalpy. In the limit of large $\mathrm{Ge}$ concentrations, i.e., close to the Ge melting temperature, the melting entropy obtained from Eq. (7) with $\Delta s$ is temperature independent, and is correct in very good approximation; i.e., $\Delta h_{m}=0.383 \mathrm{eV}$ per atom. We cannot obtain more detailed information from the data in Fig. 2(b) without more specific and independent knowledge of the temperature (or coverage) dependence of either $\Delta h_{m}$ or $\Delta s_{m}$.

The total latent heat of a droplet with a fixed number of Pt atoms, traveling from colder to a hotter position, thus increases for two reasons: (1) an increasing latent heat per atom and (2) an increasing number of Ge atoms within the droplet. As a result, the eutectic droplet experiences a strong incentive to move toward the hottest position at the surface. The effect is present all along the surface and is stronger when operating closer to the melting point of pure Ge [1]. We estimate that in our current experiments the surfed temperature range is about $50 \mathrm{~K}$ above the eutectic temperature.

The driving force per Pt atom in the droplet for motion toward hotter positions is identical and does not depend on the droplet size, since the latter scales one to one with the number of $\mathrm{Pt}$ atoms inside the droplet. We note that the (extreme) Ge-rich part of the bulk phase diagram is relevant for the current situation of a eutectic droplet in contact with a pure Ge substrate. The liquidus line represents the equilibrium between the eutectic and mainly the pure Ge solid. As argued above, the eutectic droplets all move toward the center-i.e., the highest-temperature location (cf. Fig. 1) - of the surface when thermodynamic equilibrium between the substrate and the individual spherical droplets has set in. The final ideal situation would lead to one huge droplet in the center, growing mainly due to coalescence with arriving smaller droplets. The latter move in a radial fashion toward the center, and, for geometrical reasons, the rate of coalescence is high(est) near the center. Indeed, we have identified such behavior. As evidence we add an illustrative movie (see Supplemental Material, Movie 1 [17]). A few frames obtained near the substrate center are displayed in Fig. 3. The bright objects are, irrespective of their lateral size, spherical segments (caps) of eutectic PtGe droplets. They appear bright in these PEEM images due to a, compared to their surroundings, slightly lower work function. Their wetting angle is about $18^{\circ}$ and the incident UV light is directed along the azimuth indicated by white arrows. The brightness profile of the droplets is understood in detail as described in Ref. [18]. In short, the wetting angle has been obtained from both the PEEM intensity profile of the droplet and the position of the interference fringes of the direct and the specularly reflected light on the illuminated side of the droplet. The surface morphology is quite heterogeneous as the environment around the large droplets appears featureless, in contrast to the surroundings of the small objects. The smallest objects are sessile eutectic droplets, and all the other ones move toward the large droplet residing in the center; eventually all would merge and finally constitute a huge eutectic
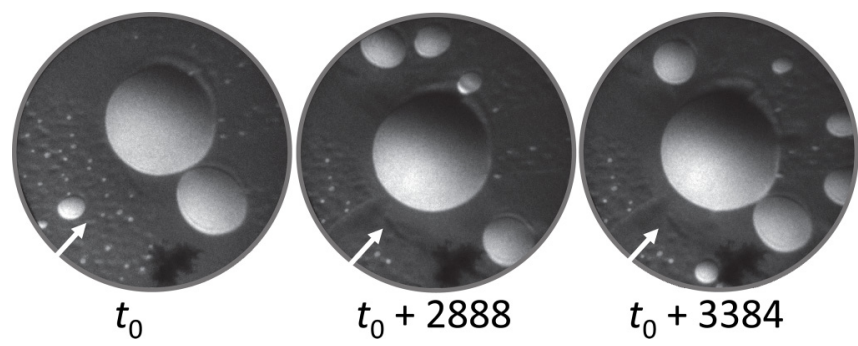

FIG. 3. Snapshots of PEEM movie (1100 K) [16], taken close to the center of the surface at an advanced stage during in a thermal steady state situation, at different points in time after the arbitrary point in time in the right-hand image (time in s). Field of view (FoV) is $150 \mu \mathrm{m}$. The bright eutectic droplets are illuminated along the direction indicated by the white arrows. They represent spherical caps with a wetting angle of $18^{\circ}$ [18]. The dark feature near the bottom is a defect in the channel plate. 


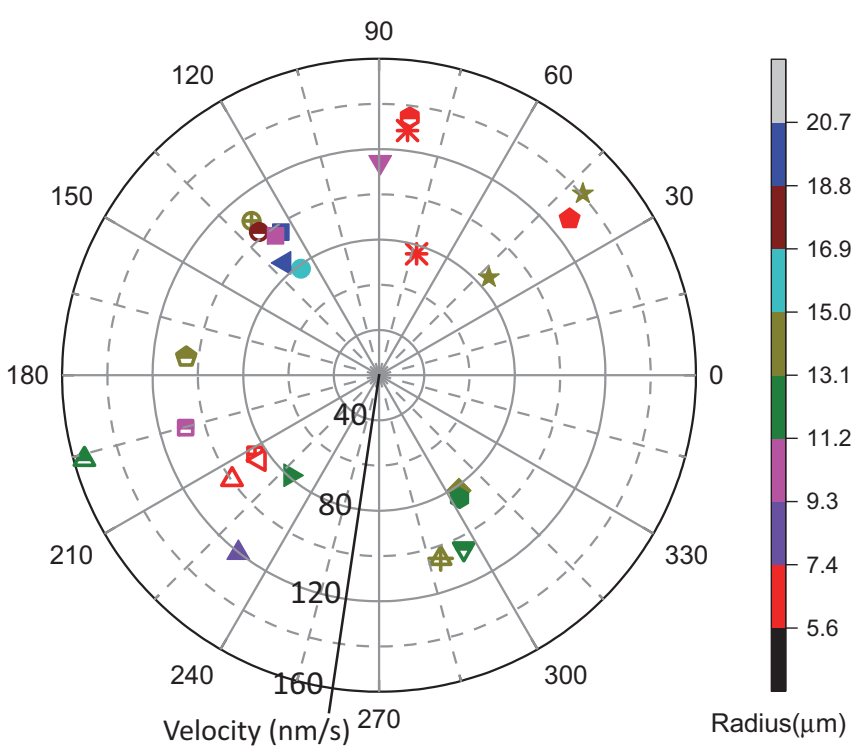

FIG. 4. Polar diagram of the moving droplets. The radius denotes their velocity in $\mathrm{nm} / \mathrm{s}$ (left scale) and the (arbitrary) azimuth their direction of motion. A given point on the negative horizontal axis would thus represent a droplet traveling from right to left toward the hottest spot. The colors refer to the droplet size (right scale). The substrate temperature was $1100 \mathrm{~K}$. Each symbol represents a different droplet.

droplet with a Ge content that corresponds to the reigning temperature and given by the liquidus line in Fig. 1. It is estimated that droplets with a diameter less than about 2-3 $\mu \mathrm{m}$ remain immobile under the current conditions. The latter behavior was observed previously for the related $\mathrm{PtSi} / \mathrm{Si}$ (100) system also by Yang et al. [4].

Next, we consider eventual crystalline preferences for the direction of motion for the moving droplets. The result for some 30 eutectic droplets is summarized in Fig. 4. It exhibits a polar plot of the direction and the magnitude of the velocity of the moving droplets. Any data point situated in the first quadrant represents a motion from globally bottom left to the center at a velocity given by the radius (left-hand scale in $\mathrm{nm} / \mathrm{s}$ ). The colors represent the size of the objects (right-hand scale, radius in $\mu \mathrm{m}$ ). The final large droplet and the sessile small droplets are not included in the plot.

The data do not reveal any correspondence between the velocity and the droplet size. Moreover, the data show no preference for any azimuth at this surface with its two-dimensional symmetry. These observations lead unequivocally to two conclusions: (1) The motion is driven solely by the direction of the local temperature gradient and (2) the twofold crystalline anisotropy plays no role. These facts both disqualify the anisotropy of Ge dissolution kinetics at atomic steps as the driving force for the droplet motion as was suggested in Ref. [19] for the motion of AuSi droplets on Si(111). In addition, it is noted that the obtained velocities of roughly $70-130 \mathrm{~nm} / \mathrm{s}$ are right in the ballpark of literature values found for moving eutectic droplets on varies surfaces [4-6,8$10,19,20]$ and, further, that no distinct relation between the size and the velocity is observed. The latter is in line with several earlier reports too [4-6].
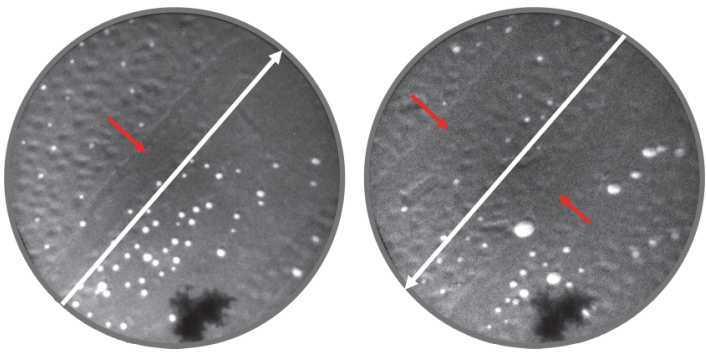

FIG. 5. Snapshots from a movie representative of the motion of droplets "far" away from the center. In both images the manipulator was moved in such a way that the center moved out of the field of view along the direction of the white arrow, in the left-hand case by almost $1 \mathrm{~mm}$ and in the right-hand case by about $0.5 \mathrm{~mm}$. Small sessile droplets reside in areas that look quite heterogeneous. Note that in PEEM one images the local work function, and the heterogeneity could be caused by either roughness, composition, or a combination of both. The larger droplets still move under the influence of a thermal gradient and move along the direction of the arrows. They leave behind trails with a much more homogeneous appearance and a few are marked with red arrows. Moving droplets have a strong tendency to follow fresh trails. This can also be observed in the first movie [17]. The field of view is 150 microns, and the temperature is $1100 \mathrm{~K}$.

The motion of droplets driven by the thermal gradient is highly complex. This is caused in part by the lack of homogeneity on the surface as is apparent from Figs. 3 and 5. In the left- and right-hand panels of Fig. 5 the field of view is displaced by, respectively, about 1 and $0.5 \mathrm{~mm}$ in the direction opposite to that of the white arrows. The sessile small droplets with a diameter of about $2-3 \mu \mathrm{m}$ or less remain immobile in an environment with a heterogeneous work function as concluded from the varying local brightness. Note that in PEEM the contrast results predominantly from differences in work function and not purely from height variations. The motion is oriented toward the center; i.e., it follows a positive local temperature gradient to the maximum at the center. The "wakes" of the passing droplets (a few are indicated by red arrows) appear substantially more homogeneous when compared to their environment. Also, a strong tendency to follow the wakes of earlier passing droplets is observed. This happens at a higher velocity than that of the pioneering droplets distinctly more slowly finding their way through the heterogeneous areas. This behavior is also apparent in the movie (Supplemental Material, Movie 1 [17], underlying Figs. 3 and 4.

The actual situation may even be further complicated by leftover traces from previous experiments. An illustration is shown in Fig. 6, where the currently prevailing motion is from left to right at an angle of about $-30^{\circ}$. A remainder of a trail (wake) of a moving droplet in an earlier experiment is pointed at by the dashed red arrow. We cannot make a conclusion on its direction of motion: It was probably from left to right but could have been from right to left.

Obviously again, the motion of the eutectic droplet is a complicated process, which is, moreover, hard to define exactly due to heterogeneities at the surface, which are inherent to the (current and previous) Pt deposition and heating experiments. That fact prevents us from performing systematic 


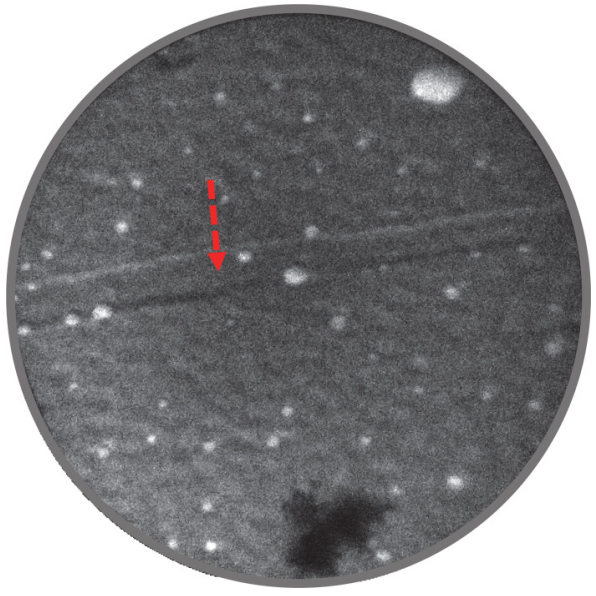

FIG. 6. A snapshot from a different position at the surface, in this case about a few tenths of a millimeter left of the center. The dashed arrow refers to a trace left from an earlier experiment (see text).

temperature dependent measurements of the velocity to gain information on activation energies.

We emphasize here that the speed of the moving droplets does not depend on the magnitude of the local temperature gradient. This finding is highly relevant and in line with the results of studies of similar systems [5] and will be treated in detail further below when discussing the data in Fig. 13.

Almost all studies dealing with the velocity of eutectic droplets in the presence of a temperature gradient adopt the bulk-based model put forward by Cline and Anthony [1]. They find for the migration velocity $v(T)$ of a liquid eutectic droplet at a temperature $T$ the following expression:

$$
v(T)=\frac{D(T)}{1-X(T)}\left(\frac{\partial X}{\partial T}\right) \nabla T,
$$

with $D(T)$ the diffusivity of the host material (in our case $\mathrm{Ge}$ ) inside the droplet, $X(T)$ is the normalized alloy fraction along the liquidus line, and $\nabla T$ is the temperature gradient. $X(T)$ approaches 1 when the guest component (in our case Pt) inside the droplet becomes negligible compared to the host component. The migration velocity was found about constant even if the volume of the droplets is changed by three orders of magnitude. In other words, the migration speed of the eutectic droplets does not depend on the size, which is probably the reason for the surprising and remarkable popularity of this bulk model for explaining migration rates of the eutectic droplets on surfaces. Assuming that this model indeed holds for droplets on surfaces too, we note that a decrease of $\nabla T$ near the center goes along with a higher $X(T)$ value. In all cases we stay well below the melting temperature and a variation of $1-X(T)$ by two orders is way off limits. The independence of the velocity of the moving droplets on the thermal gradient as noted in the previous paragraph remains to be understood.

We now address the motion of single droplets as well as their mergence in much more detail. In the current experiment a large droplet moves from left to right in Fig. 7. It does so slowly, because it is held up while "consuming" all the smaller, sessile droplets on its way. A movie is added to illustrate this behavior (see Supplemental Material, Movie 2

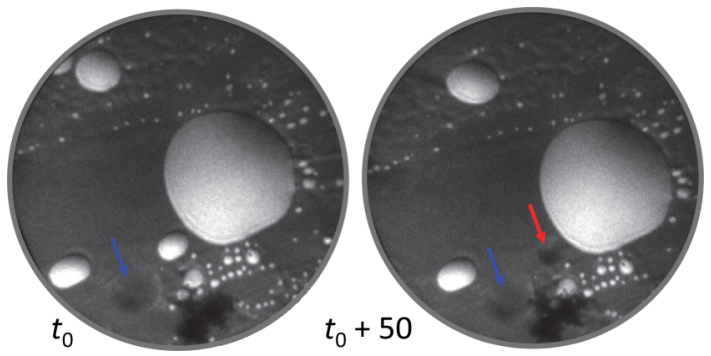

FIG. 7. PEEM images from a movie $(1100 \mathrm{~K})$ in a different experiment on another $\mathrm{Ge}(110)$ surface; FoV is $150 \mu \mathrm{m}$. The images show evidence for the remainder of the trails from moving droplets in a previous experiment. This is the reason for the appearance of sets of small droplets heading from left to right. Their alignment is caused by the sweep of passing droplets in an earlier experiment. We observe several trails. Left: just before the coalescence of a small droplet with the large one. Right: just after coalescence with the red arrow marking a left-behind footprint. The blue arrows mark a "footprint" left behind in an earlier coalescence event.

[17]). The wake of this huge droplet appears remarkably homogeneous, i.e., much more so than is the case in the wider surroundings. The same is true for the trails left behind by the smaller moving droplets without exception. A similar observation was reported earlier $[4,6]$ for motion of eutectic droplets in similar systems. To rationalize this observation one may assume that the eutectic droplets move on nonreconstructed areas of the $\mathrm{Ge}(110)$ surface. Note that for the clean surface, i.e., in the absence of deposited $\mathrm{Pt}$, the $(1 \times$ 1) structure is observed at the elevated temperature, $1100 \mathrm{~K}$, maintained here. This is evidenced by Fig. 8(a), which shows the diffraction pattern taken with $30 \mathrm{eV}$ electrons with the extinct peaks $(0,1)$ and $(0,-1)$ characteristic of the $(1 \times 1)$ pattern of a (110) surface of a diamond crystal such as Ge(110) (see also Fig. 9). Instead, we observe for an area, selected outside the "footprint" of the droplet in Fig. 7 (see caption) the diffraction pattern exhibited in Fig. 8(d). It represents a $(2 \times 1)$ structure as sketched in Fig. 9(c) with its corresponding diffraction pattern in Fig. 9(d).

Here every second atomic string of one of the two Ge sublattices is replaced by a string of Pt atoms (sketched in red). The replacement of each $\mathrm{Ge}$ atom in that string leads to noncomplete extinction of the $(0,1)$ peak (in a single domain) due to the different scattering cross sections for Pt and Ge. The constructed $(2 \times 1)$ pattern matches the one observed in Fig. 8(d), and we thus conclude that the uniform wake of the moving eutectic droplets consists of $(2 \times 1)$ cells with a composition of one $\mathrm{Pt}$ atom per three Ge atoms. This ratio $(0.25)$ is close to that $(0.22)$ at the eutectic point. We note that no preference for the Pt exists for the replacement of $[-110] \mathrm{Ge}$ chains belonging to either the bluish or the reddish sublattice. Therefore, different equivalent domains coexist. The size of the domains must be small compared to the transfer width of the instrument used (several tens of $\mathrm{nm}$ ) as we conclude from the extinct $(0, \pm 1)$ peaks in the $(2 \times 1)$ diffraction pattern. The distance between two neighboring [-110] Pt chains is $1.12 \mathrm{~nm}$, i.e., exactly equal to the minimum distance between Pt induced nanowires [21]. 


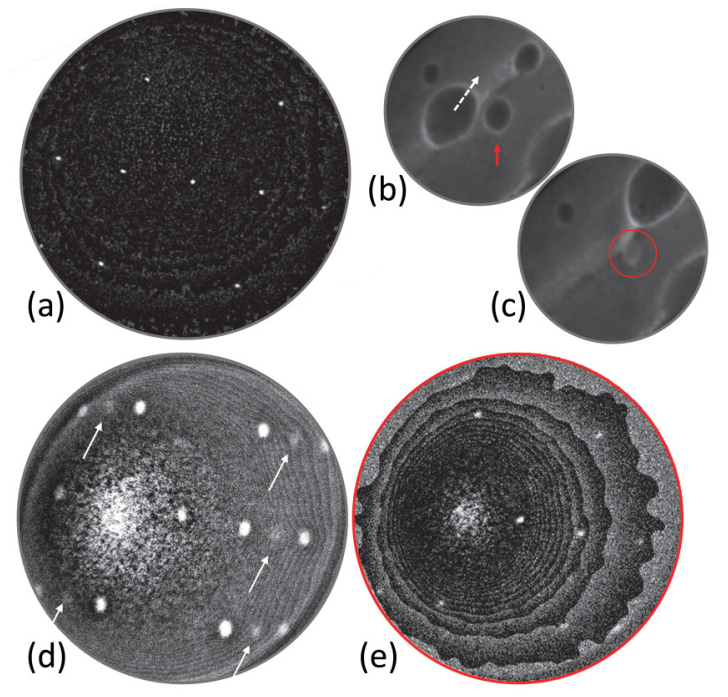

FIG. 8. (a) Diffraction pattern of the clean surface measured at $1100 \mathrm{~K}$ and taken at $30 \mathrm{eV}$. (b), (c) Mirror image data (Start voltage $-1.3 \mathrm{eV}$ ) from the footprint area, respectively, before and after coalescence of the droplets in the center resulting in the encircled footprint in (c) of the droplet pointed out in (b) by the red arrow. The white dashed arrow indicates the direction of motion of the larger droplet, which has advanced to the edge in (c). FoV is $15 \mu \mathrm{m}$. N.B. The images were taken in mirror mode. Note the reversal of contrast when compared to PEEM. (d), (e) diffraction patterns taken at $30 \mathrm{eV}$ outside and inside the footprint, respectively. The arrows indicate a few half-order peaks revealing a $(2 \times 1)$ reconstruction in $(d)$.

We now return to Fig. 7. Smaller droplets follow the thermal gradient in the wake of the large one. They are about equally fast, and all eventually catch up with the large one resulting in successive mergences of the small one(s) with the large one. The large droplet has a straight contact line with the surface because it touches and wets a step bunch (bottom-right side). The main part of the subsequently arriving droplets follows its wake and they tend to make contact to the step bunch too. The two panels illustrate a mergence event of a smaller droplet with the largest one. It is highly remarkable and meaningful that the smaller of the merging droplets leaves behind a "footprint", indicated by the red arrow. This footprint is an area with a higher work function since it is imaged at a lower brightness in PEEM. The blue arrow shows a similar footprint after a previous mergence event. All footprints are remarkably stable with time even at the current high temperatures. Using spatially resolved low-energy electron diffraction ( $\mu$ LEED) we have also measured diffraction patterns inside these areas. An example is shown in Fig. 8(e). Here the diffraction pattern is measured inside the footprint of the smaller droplet after coalescence in Fig. 8(c). We note in passing that the brightness in mirror images of the footprints is higher than their environment in contrast to PEEM images. This is in both cases a consistent signature of the higher work function in the footprints. To our surprise we find a $(1 \times 1)$ diffraction pattern [Fig. 8(e)], which is indicative of a nonreconstructed $\mathrm{Ge}(110)$ patch. Evidently, the footprint is swept clean from previously present Pt during coalescence and a direct consequence is that the droplets make direct contact with the underlying substrate.

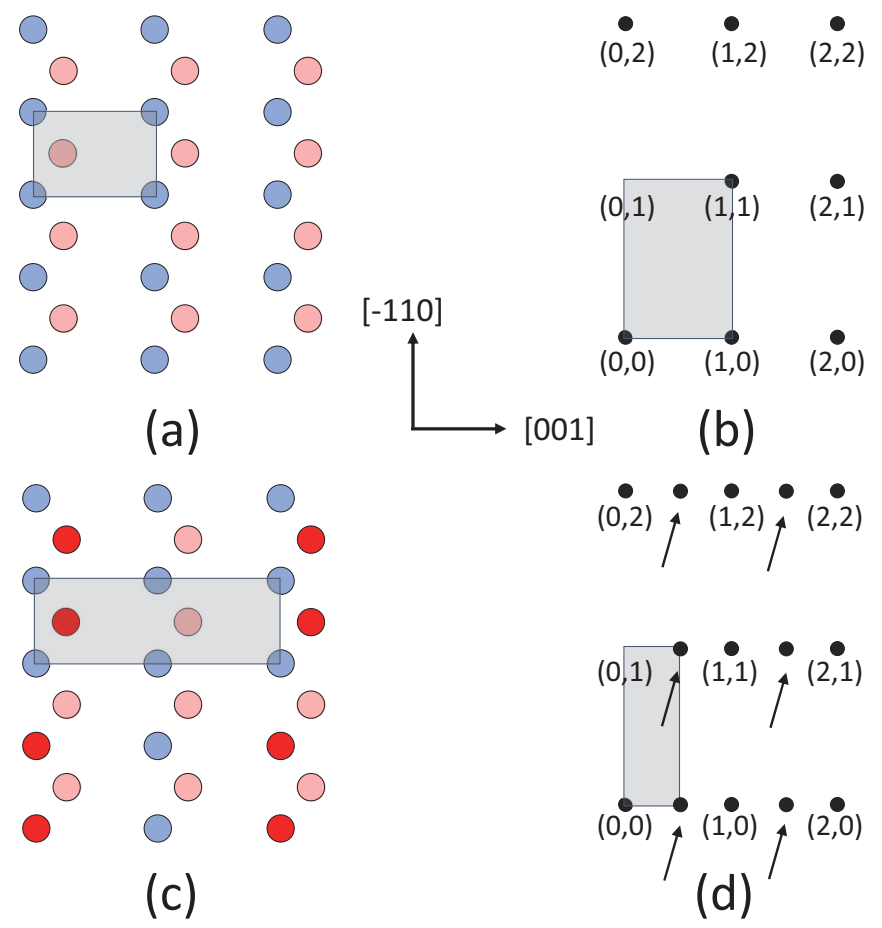

FIG. 9. The crystal directions are indicated and the two identical sublattices are distinguished by a different color. (a) The atomic arrangement of atoms in the uppermost layer of a bulk terminated (110) face, also showing a $(1 \times 1)$ unit cell; (b) the corresponding schematic LEED pattern. Note the extinct $(0,1)$ peak as a direct consequence of the diamond structure of $\mathrm{Ge}$; (c) each second of the $[-110]$ surface strings of the reddish sublattice is replaced by $\mathrm{Pt}$ atoms (red). A possible $(2 \times 1)$ unit cell is shown too. An equivalent domain with exchange of $[-110]$ strings of the bluish sublattice is possible and involves a glide line. $(\mathrm{d})$ Corresponding $(2 \times 1)$ LEED pattern. For the sketched configuration the $(0,1)$ peak is also extinct if the domains separated by glide lines are small. The glide vector runs along [-111] with a length of $\sqrt{ } 6 \AA$.

This enables a direct exchange of Ge between the substrate and the eutectic droplet on its way to areas at higher temperatures in order to obey the temperature-imposed composition, i.e., the Ge-Pt ratio on the liquidus line. The mass exchange takes place readily via atomic steps, multiple steps, or step bunches. At the same time this explains how substrate steps are affected by the passage of a eutectic droplet as observed in many publications $[5,6,8,19]$. We emphasize that even for a misalignment of $0.1^{\circ}$ the step density is of the order of 20 per running micron and steps are thus readily available for micron-sized droplets in order to establish equilibrium between the droplet and the substrate.

The overall picture that emerges so far is summarized by the sketch in Fig. 10.

A close inspection of the wake of the eutectic droplets appears to indicate that upon each passage of a eutectic droplet the trail gets slightly less bright. This is attributed to a minimal loss of Pt from the strings to the passing spherical segment of the eutectic droplet. This would result more and more in a situation that slowly approaches the pure Ge case and goes along with an increase of the work function. This would 

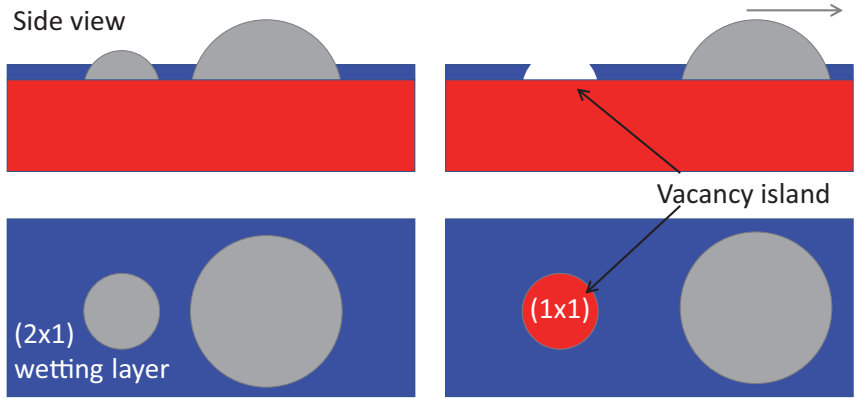

Top view

FIG. 10. Sketch of two eutectic PtGe droplets in light blue before and after coalescence on the left-hand and right-hand sides, respectively, in side view and in top view. The Ge (110) substrate is in red, the $(2 \times 1)$ wetting layer is in dark blue, and $(1 \times 1) \mathrm{Ge}(110)$ is visible in the vacancy island left behind by the smaller of the two initial droplets. The wetting layer is 1 atomic layer thick and thus much thinner than it appears here.

be accompanied by a slight increase of the average distance between the Pt strings (see Fig. 9).

Subsequently, the motion of droplets along the wake of the large one in Fig. 7 is evaluated in more detail. Characteristic and highly instructive data are exhibited in Fig. 11. A sequence of smaller droplets follows the route alongside the step bunch. A vacancy island is marked with " $n$," a consecutive droplet by " $n+1$ " [Fig. 11(a)].

In fact, " $n$ " rather marks the stable footprint of droplet $n$ which emerged during its coalescence with the large one. The red line in Fig. 11(a) indicates the trajectory followed by the small droplets. In the central part the droplets touch the step bunch at the substrate and move forward parallel to it. Figure 11(b) shows the brightness (see color scale on the right) measured along the trajectory. The corresponding position is plotted along the $Y$ axis. The time advances along the $X$ axis. The vacancy island is visible and immobile until ca $t=480 \mathrm{~s}$.
The receding edge of the large droplet is visible in the upper part of the figure and its small positive slope reveals a low speed of the order of a few $\mathrm{nm} / \mathrm{s}$. Also, the motion of droplets $n+1$ and the next one is visible and from their slope one extracts a speed of the order $100 \mathrm{~nm} / \mathrm{s}$. Upon passage of the droplet $n+1$ the position and shape of the vacancy island $n$ is affected.

Most importantly one can follow the speed of the receding edge of droplet $n+1$ during its mergence with the big droplet. This speed, which is controlled by the diffusivity of the liquid material in the small droplet during its pickup by the large droplet, is determined from the slope of the low- to high-brightness transition during the final few seconds of the data. Due to its prime importance to this paper, we illustrate these in Fig. 11(c), which shows on the left the last few frames of the movie already discussed above just before mergence of the small droplet $n+1$ with the large one on the right. The corresponding part of the brightness diagram is shown in the right-hand panel. The position of the receding edge of droplet $n+1$ is enhanced by the red lines. Just near the right edge a vacancy island emerges as the result of the mergence. During merging, the contact lines disappear between the droplets and the breaking of bonds is no longer rate limiting. The advance rate of the receding edge during mergence is not less than about 50 times faster than during the motion of the small droplet. From this strong mismatch it is immediately clear that the diffusivity inside the droplet is not the rate limiting factor for the speed of the thermally driven motion of the eutectic droplets. This is at variance with the model for bulk diffusion of eutectic droplets put forward by Cline and Antony [1] given by Eq. (8), which has been embraced in so many surface-diffusion studies. This fact, in combination with the total absence of a dependence of the speed for droplets with equal size on the magnitude of the thermal gradient, as noted further above, leads to a complete failure of Eq. (8) to describe the droplets' speed. An explanation for the velocity of the droplets must include these facts as well as the lack of influence of the droplet size. A viable scenario would then
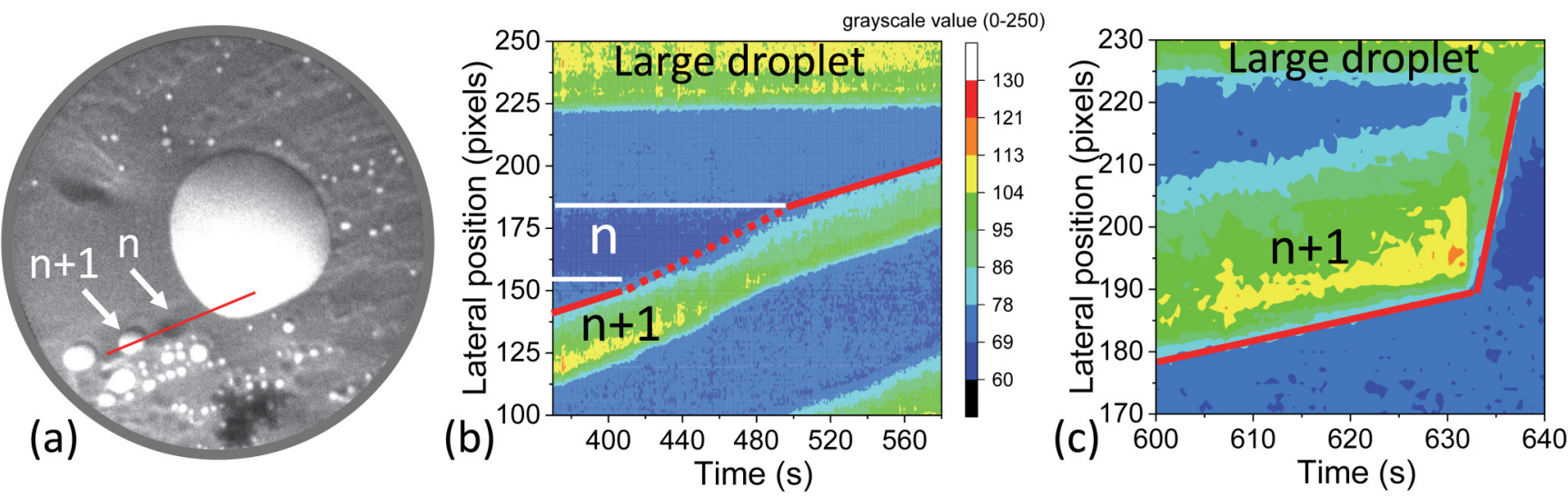

FIG. 11. (a) Frame of the PEEM movie already addressed in Fig. 7 (see that caption for details). The frame has been captured about 17 min later than the last captured frame in Fig. 7. $n$ marks the footprint of droplet $n$ 's mergence with the large one on the right and $n+1$ marks a consecutive droplet. (b) The brightness of the image measured along the red line in (a) with proceeding time. The passage of droplet $n+1$ through the vacancy cluster $n$ is highlighted. The slope of the red lines defines the velocity of the droplet before and after (solid lines) and during (dashed line) passing vacancy $n$. (c) The brightness of the image measured along the red line in (a) just before mergence of droplet $n+1$ with the large one on the right. The evolution of the receding edge of " $n+1$ " is indicated by the red lines. 
be the dissolution of the edges of the wetting layer at the advancing side (see the sketch in Fig. 10). At the receding edge the material will be incorporated in the reestablished edge. Note that the $\mathrm{Pt} / \mathrm{Ge}$ ratio in the wetting layer (1/4) and that inside the eutectic droplet are highly similar. One element in the motion of the droplet would then be dissolution of chains of atoms in the leading edge and simultaneous expulsion at the trailing edge. This process is size independent since the dissolution rate at the edge hardly depends on the radius for large droplets. It also explains the lack of dependence on the local thermal gradient since only the absolute temperature counts and thermal differences are very small anyway. If we start from a typical diffusion speed $v$ for the droplets of $100 \mathrm{~nm} / \mathrm{s}$ and rate limitation by the breaking up of atomic bonds in the edge (contact line) of the wetting layer at the advancing side of the droplet we can derive the corresponding activation barrier.

$$
v=f a \exp \left(-\frac{E}{k_{B} T}\right) \quad \text { or } \quad E=-k_{B} T \ln \frac{v}{f a} .
$$

The lattice distance is denoted by $a$. By assuming an attempt frequency $f$ of $10^{13} \mathrm{~s}^{-1}$ we arrive at $E \sim 2.2 \mathrm{eV}$.

A still unanswered question is why the small droplets (diameter less than ca. $3 \mu \mathrm{m}$ ) stay immobile across the entire surface. This appears connected to the unusual ripening behavior. It is well known due to Gibbs-Thomson that the equilibrium pressure to maintain droplets or crystallites increases with decreasing radius [22]. As a result, near equilibrium larger droplets grow at the expense of smaller ones and this process is known as Ostwald ripening. Less broadly realized is the fact that the same Gibbs-Thomson principle leads to the opposite result for vacancy clusters in the bulk or, for the same reason, vacancy clusters in two dimensions (2D). The equilibrium pressure $P_{r}$ to maintain liquid clusters in crystalline surroundings decreases with decreasing radius $r$ according to

$$
\frac{P_{r}}{P_{\infty}}=\exp \left[-\frac{2 \sigma v_{l}}{r k_{B} T}\right]
$$

i.e., smaller vacancy clusters are more stable than their larger counterparts. The interface tension is $\sigma$ and $v_{l}$ is the atomic volume in the liquid, while $P_{\infty}$ denotes the equilibrium pressure for a flat infinitely large separation plane. A similar expression holds for a liquid surrounded by a crystal in two dimensions as is the case here. In other words, it becomes increasingly difficult to dissolve edge atoms of smaller vacancy clusters in the wetting layer into the droplet. We attribute the observation of a threshold size for small droplets to become mobile in a thermal gradient to this feature.

Ripening on the surface takes place predominantly through the coalescence of moving droplets (Smoluchowski ripening) as documented above; this conclusion was also reached in previous reports [4-7]. This statement holds, in particular, in regions near the temperature maximum since, by its nature, there the density of radially moving droplets is highest and, consequently, the collision frequency is highest too. At larger distances from the center the rate of Smoluchowskitype ripening events is lower. At these positions Ostwald type ripening is also active as illustrated in Fig. 12. It shows a frame from the movie referred to earlier (Supplemental Ma-

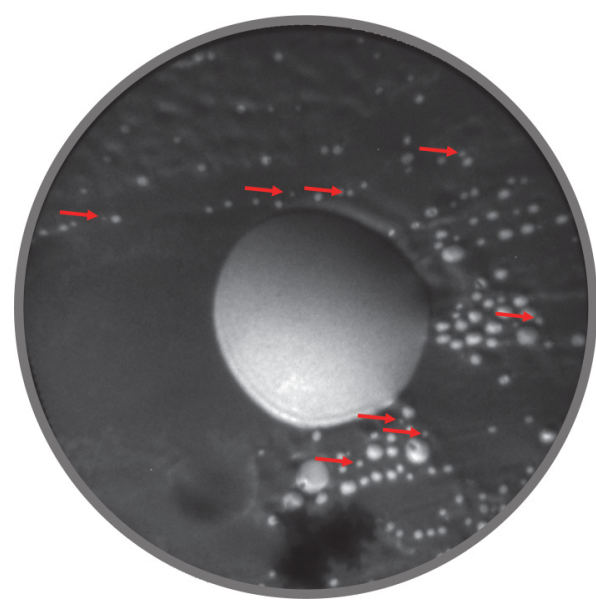

FIG. 12. Frame from the PEEM movie referred to previously (see Figs. 7 and 11). Field of view is $150 \mu \mathrm{m}, T=1100 \mathrm{~K}$. The central droplet moves to the right, while snapping smaller ones on its way to the center (not shown here). Within 47 min the small droplets, indicated by red arrows, disappear due to Ostwald ripening (see text).

terial, Movie 1 [17]) and the red arrows mark small droplets which disappear during a time lapse of $47 \mathrm{~min}$. (We cannot exclude that the involved entities are crystalline Pt containing features.) The mass transport takes place on top of the wetting layer and $\mathrm{Pt}_{x} \mathrm{Ge}_{y}$ particles are detached from the droplet at the contact line with the wetting layer. Consequently, Ostwaldtype ripening does occur, but Smoluchowski type becomes increasingly more dominant at smaller distances from the center.

Finally, we focus on the question of whether the interface or the contact plane between the droplet and the substrate is flat or has a pitlike structure. The latter has been suggested for similar systems $[4,8,10]$. A complicating factor is that the optical impression of the footprints [see Fig. 8(f)] seems to confirm that. However, it must be realized that the PEEM images only contain information on the local "structure" of the work function and thus reveal no direct information on the contour lines at the surface. Ex situ information from AFM (atomic force microscopy) or TEM (transmission electron microscopy) is useless in this case since any temperature variation necessarily leads to huge mass exchange between the eutectic droplet and the surface. N.B., in the case of cooling from, say, $1100 \mathrm{~K}$ to the eutectic temperature of $1050 \mathrm{~K}$ per Pt atom $1.2 \mathrm{Ge}$ atoms are redeposited at the surface. For a droplet with an exposed area of $10 \mu \mathrm{m}^{2}$ this implies $>1000 \mathrm{Ge}$ atoms per Ge (110) unit cell of the initial interface. Thus, extreme caution must be exercised when trying to gain information on the flatness of the eutectic droplet-substrate interface from $e x$ situ data. Therefore, one is thrown back to indirect methods to shed light on this issue. In an attempt we presume that endotaxy applies and instead of a monolayer-thick vacancy cluster in the wetting layer, the footprint marked with $n$ in Fig. 11(a), one then deals with a huge, thousands of layers deep pit. The passage of droplet $n+1$ would accordingly take breaking up of the order of $10^{12}$ substrate atoms; one has to expect that the motion of the droplet must be decelerated substantially. Instead, it is found that the passage of the footprint $n$ by droplets leads to a temporary acceleration of the velocity of the droplet by about 30\% [see Fig. 11(b)]. We 


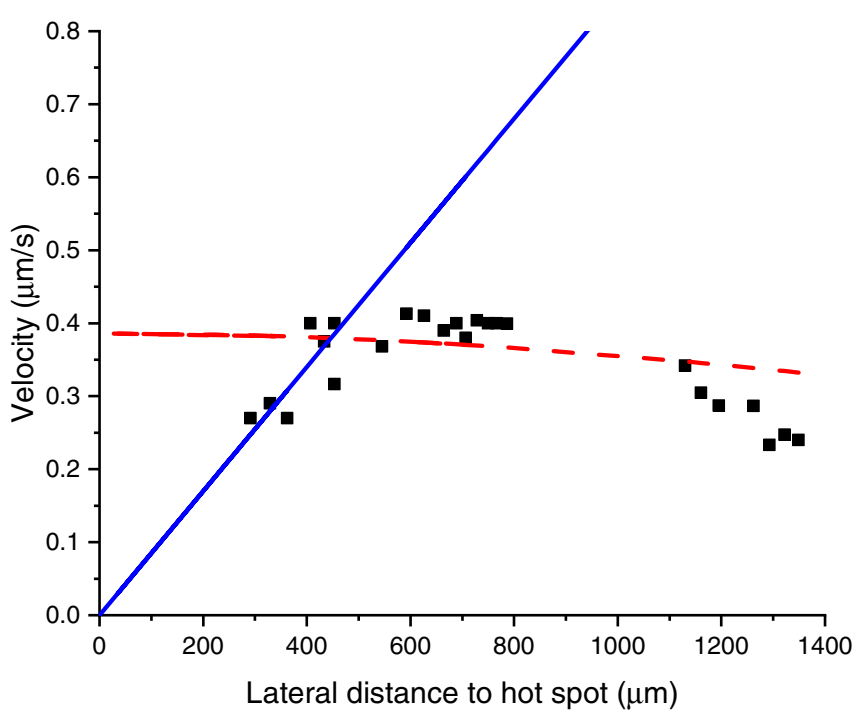

FIG. 13. Velocity in $\mu \mathrm{m} / \mathrm{s}$ of a moving droplet followed from 1200 to $200 \mu \mathrm{m}$ toward the center (black squares). Between ca. 750 and $1100 \mu \mathrm{m}$ the droplet follows a preexisting trail at an enhanced speed. The straight blue line from the origin through the cloud of the first seven data points indicates the expected behavior for a paraboloid-shaped temperature profile on the basis of Eq. (8). See text for further details. Note that the velocity values are somewhat higher than those in Fig. 4, which is attributed to a nonreversible change in the thermocouple, which caused an offset of about $20 \mathrm{~K}$.

conclude that this finding is hard to reconcile with any sizable pit (endotaxy) underneath the eutectic droplet. In contrast, the acceleration is understood straightforwardly with the vacancy cluster model: Passage of the vacancy cluster involves the breaking of lesser bonds at the edge of the vacancy cluster and thus locally speeds up the motion.

\section{DISCUSSION AND CONCLUSION}

A widespread notion for thermomigration of micron-sized eutectic droplets assumes that the driving force is given by the relative rates of dissolution of substrate material at the advancing side and redeposition at the receding side. A concentration gradient of host substrate material (in the current case Ge) is maintained by its low diffusivity inside the eutectic droplet, which is finally limiting the speed of the moving droplets. This model provides a convenient way to explain the widely observed size independence of this speed "since driving force and mobility scale inversely" [5]. We think that this idea has contributed largely to the success of this model. Our current study for migration of Ge-Pt eutectic droplets on $\mathrm{Ge}(110)$ demonstrates that the explanation is in contrast with some key observations. First, and of prime importance: The diffusivity inside the droplet is more than an order of magnitude faster than needed to make diffusion of Ge inside the droplets rate limiting for their speed. Second, and equally significant, is the fact that the speed of migrating droplets with equal size is unaffected by a thermal gradient that varies by as much as a factor of 6 (see Fig. 13). Obviously, in this situation inverse scaling of mobility and driving force does not apply. In order to access this information, we monitored the speed of a moving droplet from about $1.2 \mathrm{~mm}$ from the temperature maximum toward about $0.2 \mathrm{~mm}$ from the hottest spot. The results are exhibited in Fig. 13 given by the black squares. The variation of the absolute temperature at the visited positions is about $7 \mathrm{~K}$ and the rate of thermally activated processes is thus virtually constant. The red curve (dashes) shows the variation for an actual activation energy of $2.2 \mathrm{eV}$.

The current model predicts according to Eq. (8) a linear variation with the thermal gradient. An estimate is illustrated by the straight blue line through the origin in Fig. 13. Within the position window, in which data were gathered, one should expect an increase of the velocity by a factor of not less than 6. Instead, the velocity remains constant or even leans toward a minor decrease. The result thus reveals an obvious failure of the currently popular model. To explain our observation, we instead propose that the droplets make direct contact with the substrate and are surrounded by a flat wetting layer. The rate limiting step for the migration speed is then determined by the rate of dissolution in the contact line of the wetting layer with the moving droplet at the advancing side. The integrity of the wetting layer is restored at the receding side. The involved activation energy amounts to $2.2 \mathrm{eV}$, assuming a frequency factor of $10^{13} \mathrm{~Hz}$. A very slight increase of $\mathrm{Ge}$ per $\mathrm{Pt}$ atom content within the droplet during travel is readily provided by exchange at preexisting substrate steps. The interface between droplet and substrate remains basically flat. This model naturally provides the explanation for the size independence of the motion and also for its independence of the thermal gradient. The presence of a wetting layer is also held responsible for the relative smoothness in the wake of the moving droplets.

Our model also offers an alternative and quantitative description for the temperature dependence of the velocity of traveling eutectic droplets that appeared in the most detailed reports in literature so far. The result for $\mathrm{Au}-\mathrm{Ge}$ droplets on Ge(110) [14] is exhibited in Fig. 14. The experimental results have been taken from Ref. [9] (Fig. 5) and the calculated mobility curve by the dashed curve from the bulk-based model. The blue dots are obtained with our model with an activation energy of $1.75 \mathrm{eV}$. The obtained frequency factor is $\sim 10^{11} \mathrm{~Hz}$. The latter description provides a clearly convincing result. Note that the three experimental points around $1010 \mathrm{~K}$ deviate severely from the overall trend for unknown reasons.

Another direct support of our current model is illustrated in Fig. 15 for the motion of PtSi eutectic droplets on $\mathrm{Si}(100)$. The beautiful experimental data have been taken from Ref. [4] (Fig. 7). Both the temperature dependence and the remarkable temperature dependent critical size for motion of droplets have remained unexplained so far. It is even in obvious contrast with a velocity limiting diffusion rate of $\mathrm{Si}$ within the droplets. Our model offers a straightforward framework for understanding the temperature dependence of the velocity of the moving droplets. An Arrhenius plot of $\ln v$ versus $T^{-1}$ yields a perfect straight line with a slope that corresponds to an activation energy of $2.0 \mathrm{eV}$ and a frequency factor of $\sim 3 \times 10^{10} \mathrm{~Hz}$. Note that the size independence is a natural aspect of our model. Even the enigma of a temperature dependent critical droplet size is easily resolved. The only assumption to make is that in line with Eq. (10) the relative pressure $P_{r} / P_{\infty}$, necessary to keep the contact line of the vacancy inside the wetting layer intact, is constant, or $\sigma / r T$ is 

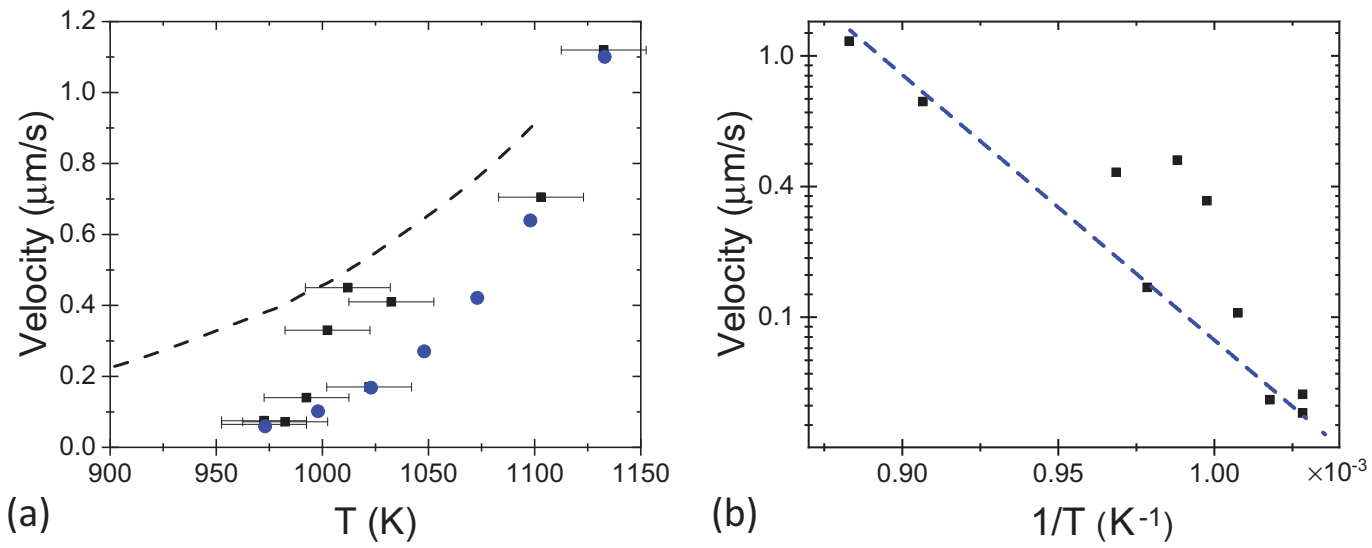

FIG. 14. (a) Measured (black squares) and calculated (dashed curve) traveling velocities of Au-Ge eutectic droplets on Ge(110) as replotted from Fig. 5 in Ref. [9]. The blue dots were obtained using our current model (see text). (b) Semilog plot of measured velocities and reciprocal temperature.

constant. The only thing to realize is that $\sigma$ varies in a generic way as a function of $T / T_{C}$ when the (twofold) symmetry of the surface is accounted for properly. We have taken the roughening temperature $T_{C}$ equal to the melting temperature but note that the exact choice is of marginal importance. With the most accurately known value at $1358 \mathrm{~K}$ as a fitting point the critical value for $r_{c}$ at the other two temperatures is calculated readily. We consider the result as highly encouraging and conclude overall that the bulk-based model for motion of eutectic droplets on a surface fails and propose instead to apply our current model. The structure of the wetting layer for PtSi on $\mathrm{Si}(100)$ is $c(4 \times 2)$ and that for $\mathrm{Au}-\mathrm{Si}$ on $\mathrm{Ge}(110)$ is $(2 \times 1)$. A careful reconsideration and reevaluation of the validity of the Cline and Anthony model for diffusion of eutectic droplets on surfaces is urgently required.

Finally, we emphasize that our current results refer to the mobility of large (micron-sized) droplets on surfaces. The nice studies by Müller and colleagues (e.g., [8,19]) address the mobility of (much) smaller droplets. The latter occurs in a different ballpark with a much larger influence of steps as mentioned already by Sutter and co-workers [5,6]. The notion that droplets move step upward would and cannot apply for the large droplets considered here. In this case and in extremity an upward self-propelling would demand the build up of a base shape of a not yet erupted "volcano" at the center of the surface, i.e., the hottest spot. This unphysical picture is at variance with our observations. It is noted as a disclaimer too that the experiments of Müller et al were performed mainly during deposition of metals on the semiconductor substrate.

The current system shows two remarkable properties, which are in seeming contradiction with a standard homoepitaxial situation of coexisting large and small adatom or vacancy islands on a surface. Usually, the smaller islands disappear causing the larger ones to grow due to either Ostwald, or to Smoluchowski ripening, or to both' cf. Ref. [23]. In this case, the small clusters survive hours, or up to several days, in the vicinity of much larger ones, even at the applied high temperature of about $1000 \mathrm{~K}$. Also, in contrast to the standard situation, e.g., [23], the small clusters stay immobile while the larger ones move under the influence of a thermal gradient. This motion is solely responsible for the observed Smoluchowski ripening in this system. We attribute both remarkable properties to a single reason: the stability of the two-dimensional contact line between the droplet and
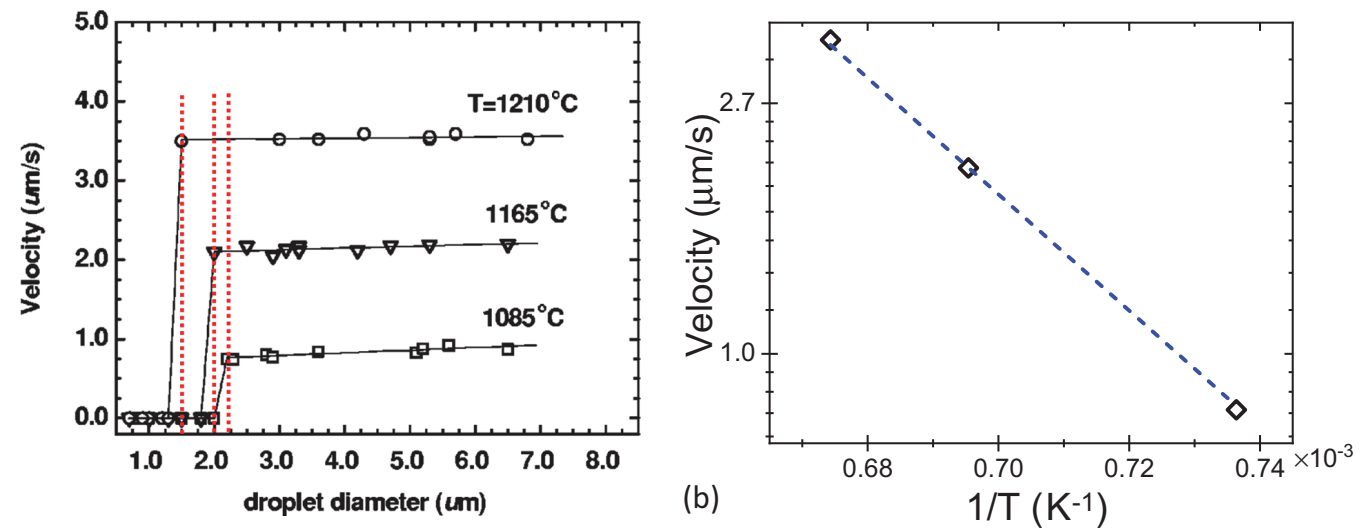

FIG. 15. (a) Velocity of moving eutectic Pt-Si droplets on $\mathrm{Si}(100)$ as a function of their diameter at three different temperatures. The red vertical lines represent the critical values for motion of the droplets (see text). The experimental data were taken from Fig. 7 of [4]. (b) Semilog plot of velocities versus reciprocal temperature. 
the PtGe wetting layer, absent in the standard situation. The Laplace pressure of PtGe inside the contact line is lower than outside and that difference increases with decreasing radius of curvature. Consequently, at any given temperature, dissolution of the contact line, as needed for either diffusion or decomposition, gets less pronounced with decreasing size.

\section{ACKNOWLEDGMENTS}

Z.Z. thanks the China Scholarship Council (CSC 201708130089) for financial support. H.J.W.Z. and A.v.H. acknowledge the Nederlandse organisatie voor Wetenschappelijk Onderzoek (NWO) for financial support.
[1] H. E. Cline and T. R. Anthony, J. Appl. Phys. 47, 2325 (1976).

[2] W.-X. Tang, C.-X. Zheng, Z.-Y. Zhou, D. E. Jesson, and J. Tersoff, IBM J. Res. Dev. 55, 10:1 (2011).

[3] J. Tersoff, D. E. Jesson, and W. X. Tang, Phys. Rev. Lett. 105, 035702 (2010).

[4] W.-C. Yang, H. Ade, and R. J. Nemanich, Phys. Rev. B 69, 045421 (2004).

[5] P. A. Bennett, J. Chobanian, J. I. Flege, E. Sutter, and P. Sutter, Phys. Rev. B 76, 125410 (2007).

[6] P. Sutter, P. A. Bennett, J. I. Flege, and E. Sutter, Phys. Rev Lett. 99, 125504 (2007).

[7] A. El-Barraj, S. Curiotto, F. Cheynis, P. Müller, and F. Leroy, Appl. Surf. Sci. 509, 144667 (2020).

[8] S. Curiotto, F. Leroy, F. Cheynis, and P. Müller, Sci. Rep. 7, 902 (2017).

[9] B. H. Stenger, A. L. Dorsett, J. H. Miller, E. M. Russell, C. A. Gabris, and S. Chiang, Ultramicroscopy 183, 72 (2017).

[10] F. Leroy, A. El Barraj, F. Cheynis, P. Müller, and S. Curiotto, Phys. Rev. Lett. 123, 176101 (2019).

[11] S. Kodambaka, J. Tersoff, M. C. Reuter, and F. M. Ross, Science 316, 729 (2007).

[12] O. Gürlü, O. A. O. Adam, H. J. W. Zandvliet, and B. Poelsema, Appl. Phys Lett. 83, 4610 (2003).

[13] H. J. W. Zandvliet, A. van Houselt, and B. Poelsema, J. Phys.: Condens. Matter 21, 474207 (2009).

[14] C. H. Mullet and S. Chiang, Surf. Sci. 621, 184 (2014).
[15] J. S. Wang, S. Jin, W. J. Zhu, H. Q. Dong, X. M. Tao, H. S. Liu, and Z. P. Jin, CALPHAD: Comput. Coupling Phase Diagrams Thermochem. 33, 561 (2009).

[16] D. A. Porter, K. E. Easterling, and M. Y. Sherif, Phase Transformations in Metals and Alloys (CRC Press, Boca Raton, FL, 2009), Chaps. 1 and 4.

[17] See Supplemental Material at http://link.aps.org/supplemental/ 10.1103/PhysRevMaterials.5.125602 for two supplementary movies: Movie $1150 \mu \mathrm{m}$ FOV movie of coalescence of eutectic droplets very close to the hot spot on the sample. The total time of the movie amounts to 6 hours and 30 minutes. The temperature is $1100 \mathrm{~K}$. Movie $2150 \mu \mathrm{m}$ FOV movie of travelling eutectic droplets towards the hot spot on the sample. The total time of the movie amounts $1305 \mathrm{~s}$. The temperature is $1100 \mathrm{~K}$.

[18] Z. Zhang, B. Poelsema, H. J. W. Zandvliet, and A. van Houselt, Phys. Rev. Materials 5, 105601 (2021).

[19] S. Curiotto, F. Leroy, F. Cheynis, and P. Müller, Surf. Sci. 632, 1 (2015).

[20] C. H. Liu, W. W. Wu, and L. J. Chen, Appl. Phys. Lett. 88, 133112 (2006).

[21] L. Zhang, P. Bampoulis, A. Safaei, H. J. W. Zandvliet, and A. van Houselt, Appl. Surf. Sci. 387 766, (2016).

[22] I. V. V. Markov, Crystal Growth for Beginners (World Scientific, Singapore, 2003).

[23] P. A. Thiel, M. Shen, D.-J. Liu, and J. W. Evans, J. Phys. Chem. C. 113, 5047 (2009). 